\title{
Radio-Acoustic Integrated Network for Ocean Information Transmission: Framework and Enabling Technologies
}

This paper was downloaded from TechRxiv (https://www.techrxiv.org).

\section{LICENSE}

CC BY 4.0

SUBMISSION DATE / POSTED DATE

$10-08-2021 / 12-08-2021$

\section{CITATION}

Chen, Fangjiong; Jiang, Zilong; Ji, Fei; Yu, Hua; Guan, Quansheng; Liu, Feng (2021): Radio-Acoustic Integrated Network for Ocean Information Transmission: Framework and Enabling Technologies. TechRxiv. Preprint. https://doi.org/10.36227/techrxiv.15139824.v1

$\mathrm{DOI}$ 


\title{
Radio-Acoustic Integrated Network for Ocean Information Transmission: Framework and Enabling Technologies
}

\author{
Fangjiong Chen, Zilong Jiang, Fei Ji, Hua Yu, Quansheng Guan, Feng Liu
}

\begin{abstract}
An important vision of next generation mobile system is to provide global internet access. The Space-Terrestrial Integrated Network(STIN) has been proposed and intensively studied to tackle this challenge. Due to the severe attenuation of radio signals in water, The STIN can not directly applied in underwater scenarios. In this paper we envision a framework of integrated radio-acoustic network arming at high-efficient data transmission in underwater scenarios, where acoustic signal is for underwater communication and radio signal is for surface and air communications. Since radio links have much higher data transmission rate and lower delay, in the integrated radioacoustic network, the acoustic links are easily become congested, at the same time the radio links are not fully utilized. We therefore propose that the integrated radio-acoustic network should be properly designed to minimize the hop count of acoustic links, as well as the signaling overhead in the acoustic subnetwork. We then presente a novel network framework and the relative technologies to help moving the signaling overhead to the radio subnetwork.
\end{abstract}

Index Terms-Integrated Network; Acoustic Communication; Network Protocols; Ocean Engineering

\section{INTRODUCTION}

The Space-Terrestrial Integrated Network (STIN) aims to guarantee the seamless coverage for billions of devices distributed over very large geographical areas and provide the global Internet access [1]. Moreover, the STIN has been coordinated with ocean network to provide network coverage on ocean surface, where the ocean network was composed of ships and other maritime platforms, which include the relay ships, ad hoc node ships and other user ships [2]. Although the integrated network had attracted intensive research, seldom attention was paid to the network coverage in ocean underwater scenarios.

Many maritime information was generated from underwater scenarios, meanwhile with various requirement of service qualities. For instance, ocean hydrology and environment monitoring require data transmission from deep ocean to water surface. Environmental monitoring data are usually not sensitive to delay. On the contrary, tsunami and underwater earthquake detection are sensitive to time delay, usually require realtime transmission. To tackle various underwater applications,

Fangjiong Chen, Zilong Jiang, Fei Ji, Hua Yu and Quansheng Guan are with the School of Electronic and Information Engineering, South China University of Technology, Guangzhou 510640, China, and also with The Key Laboratory of Marine Environmental Survey Technology and Application, Ministry of Natural Resources, Guangzhou 510300, China. Feng Liu is with College of Information Engineering, Shanghai Maritime University, Shanghai 201306 China it is necessary to extend the current space-terrestrial-ocean integrated network to underwater space to provide underwater internet access.

For communication in underwater medium, the radio signals and optical signals do not propagate through long distances as they suffer from severe path losses and scattering with high frequency, thus acoustic waves appear to be a good choice. However, the underwater acoustic links are significantly different from radio links in the aspects of low propagation speed, long transmission range, and limited bandwidth [3]. Hence, the communication techniques and networking protocols successfully applied in radio networks cannot be directly extended to acoustic networks. To provide underwater internet access, naturally it is necessary to integrate radio and acoustic networks.

In this paper we envision a framework of integrated radioacoustic network arming at providing efficient internet access in underwater scenarios. Since the radio links have much higher data transmission and lower delay than acoustic links, in integrated radio-acoustic networks the acoustic links are easily become congested while the radio links are not fully utilized. We therefore argue that the integrated radio-acoustic network should be designed to minimize the signaling overhead in acoustic links. We then discuss a novel network framework and the relative enabling technologies in physical-layer, MAClayer and network-layer to help moving the signaling overhead to the radio subnetwork.

\section{RELATED WORK}

Ocean is not only an important place for human activities such as fishery, maritime transportation, but may also become one part of human habitats in the future. As human activities in oceans continuously increase, provisioning of highperformance, cost-effective and reliable communication network services in oceans becomes an important research topic [3]. Based on the successful terrestrial wireless systems, in the past decades various space and terrestrial integrated networks have been proposed to coordinate different wireless communication protocols including satellite communication, maritime communication and terrestrial communication, to support network coverage on ocean [1], [2], [4]. Earlier researches were reviewed and summarized in [1]. More recently, Chien et al. [4] segmented the STIN framework into the perception layer, the cognition layer, and the intelligence layer, and proposed an intelligent data center based on software-defined networking 
$(\mathrm{SDN}) /$ network functions visualization (NFV) technologies. The key challenges and feasible technology to implement the new framework were also introduced. Yin et al. [2] presented a coordinated space, terrestrial and ocean network composed of three subnetworks, namely the terrestrial subnetwork, the ocean subnetwork and the space subnetwork, where the space network is mainly composed of maritime satellites and the ocean network is composed of ships and other maritime platforms.

For the networking in underwater, acoustics and laser are the most widely applied medias. The blue/-green laser can offer very high transmission rates (up to Gbps), but its transmission distance is limited (below $100 \mathrm{~m}$ ). Only the acoustic wave can propagate over long distances with up to kbps transmission rates (e.g., $15.36 \mathrm{kbps}$ over $10 \mathrm{~km} \mathrm{[5]} \mathrm{)} \mathrm{but} \mathrm{at} \mathrm{slow} \mathrm{propagation}$ speeds (e.g., $1.5 \mathrm{~km} / \mathrm{s}$ in sea water). This difference makes it impossible to directly apply communication and networking technologies well-developed for radio wireless networks in underwater environments. Hence, in the past decades many research works on underwater acoustic network were reported, mainly focusing on physical layer communication, medium access control, routing, as well as the topology control for underwater acoustic networks. These works have been reviewed by several published surveys so that a comprehensive discussion is ignored here. The interested readers may refer to [3] for more detail .

To provide seamless network coverage from underwater to ocean surface, it is important to integrate the underwater acoustic network with the ocean surface radio network. To the best of our knowledge, existing research on integrated network does not provide insightful investigation on the possible coordination of radio links and acoustic links. Generally the underwater acoustic network and the surface radio network are separated studied and only connected via buoy/ship gateway. In order to achieve more efficient and reliable transmission of marine information, it is necessary to analyze the radio link and underwater acoustic link in a unified theoretical networking framework. In [6] we first discuss and present the idea of coordinate radio-acoustic networks. However, how to deploy and operate such coordinate network is not discussed. This paper puts forward the concept of radio-acoustic integrated network, which integrates radio links and acoustic links into a unify network model. Then several technologies that enable the network operation are also presented to make it adapt to the diverse needs of marine information transmission.

\section{Framework of RAdio-Acoustic InTEgrated NETWORK}

We first consider a common communication task where an underwater node needs to send a message to another node through multiple wireless relays, e.g. in fig. 1 node 1 is the source and node 3 is the destination. Such a communication task raises in many practical applications. For instance in underwater security, one sensing node may need to wake up another remote sensing node for cooperative target tracking. It can be observed that there are two possible path, path 1: (1) $\rightarrow$ (2) $\rightarrow$ (3), and path 2: (1) $\rightarrow$ (4) $\rightarrow$ (5) $\rightarrow$ (3). Our

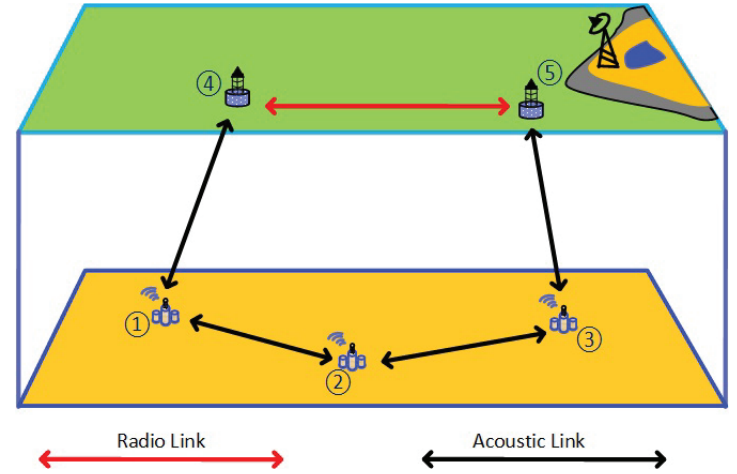

Fig. 1: Two possible paths for transforming a message from node 1 to node 3 .

motivation is that path 2 , although has more hops, may be more efficient than path 1 . More discussions are provided in the following subsection.

\section{A. Motivation}

The bandwidth of an acoustic channel is limited by ambient noise and frequency dependent attenuation. Hence the applicable bandwidth of an acoustic channel is also frequency dependent. At the transmission distance of several kilometers, the typical applicable bandwidth is $10 \sim 20 \mathrm{kHz}$ [7], which is much less than that of radio channels. To make a more specific comparison, we compare the bandwidth, data rate, latency and transmission power of typical radio communication and acoustic communication schemes at the distance of around 2 kilometers. Table 1 summarized the parameters of two typical commercialized acoustic communication modems and two standardized terrestrial wireless systems. It can be seen that the transmission rate and energy efficiency (in Watt per bps) of radio communication are about four orders higher than those of underwater acoustic communication. In general, we conclude that radio communication is much more efficient than acoustic communication.

The propagation speed of sound is about $1500 \mathrm{~m} / \mathrm{s}$ in water, which is much lower than the propagation speed of radio $\left(3 \times 10^{8} \mathrm{~m} / \mathrm{s}\right)$. The end-to-end delay of data transmission in acoustic networks can be in seconds. Hence conventional signaling exchanges based on the acknowledgement from the receivers may be inefficient due to the idle waiting time. To provide more efficient underwater internet access, it is necessary to reduce the signaling overhead or develop new signaling system for underwater acoustic networks.

Moreover, in acoustic communication, vertical transmission is more efficient than horizontal transmission. It has been shown that vertical transmission is characterized by litter time dispersion, while the horizontal channel is more easily distorted due to extremely long multipath spreads [8]. In particular, the spatiotemporal variations of the horizontal channels is much faster than that of the vertical channels, in both deep and shallow water. A simulation study shows that under same transmission distance and same bit-error-rate requirements, the transmission power of vertical transmission can be $3 \mathrm{~dB}$ lower than that of horizontal transmission [8]. 
TABLE I: Comparison of key parameters of typical radio communication and acoustic communication systems at the distance of around 2 kilometers.

\begin{tabular}{ccccc}
\hline & Evologics & Teledyne & Base Station & Wireless Bridge \\
& S2CR 15/27 & ATM-900 Series & LTE-Advanced & IEEE 802.11n \\
\hline Bandwidth & $12 \mathrm{kHz}$ & $5 \mathrm{kHz}$ & $\leq 100 \mathrm{MHz}$ & $20 / 40 \mathrm{Mhz}$ \\
Data Rate & $9.2 \mathrm{kbps}$ & $\leq 15.36 \mathrm{kbps}$ & $\leq 1 \mathrm{Gbps}$ & $72 \sim 600 \mathrm{Mbps}$ \\
Transmission Latency & $\approx 1.3 \mathrm{~s}$ & $\approx 1.3 \mathrm{~s}$ & $6.7 \mu \mathrm{s}$ & $6.7 \mu \mathrm{s}$ \\
Transmission Power & $\approx 5 \mathrm{~W}$ & $\mathrm{NA}$ & $\approx 2 \mathrm{~W}$ & $\approx 2 \mathrm{~W}$ \\
\hline
\end{tabular}

Hence, for the communication task shown in fig.1, path 2 can be more appropriate. The networking schemes of acousticradio integrated networks should be able to discover path 2 .

\section{B. Principle}

For an integrated network architecture consisting of radio links and acoustic links, there is a common situation where the acoustic links are fully occupied while the radio links are idle. The acoustic links are the bottleneck of the whole network. We argue that the signaling exchange in the acoustic links should be limited and the data flow should be directed to radio links as far as possible. The network protocols of integrated radioacoustic network should be designed by taking into account the following principles

1) Minimize the signaling overhead of acoustic links. This is to reduce the signaling traffic over the acoustic links and save the capacity for pure data traffic.

2) Minimize the hop counts of acoustic transmission. Due to the slow propagation speed of acoustic signal, wireless multihops routing in underwater acoustic network require a long period to set up the proper path. Existing research show that network throughput significantly decreases as the hop account increases.

\section{Framework}

Based on the above discussion, we propose the radioacoustic integrated network framework shown in fig.2. There are three types of communication nodes in the network. 1) Underwater communication nodes that are equipped with acoustic communication device. 2) Ocean surface nodes that are equipped with both radio and acoustic communication devices. 3) Space/air/terestrical nodes that are equipped with radio communication devices.

The network framework has the following features.

1) The radio subnetwork is fully connected. That is, any radio communication device can be connected with any other radio device via a proper routing procedure.

2) The radio subnetwork has a processing center to coordinate all the radio devices.

3) The surface node can simultaneously process the radio device and the acoustic communication device.

4) The underwater node can reach the surface node via onehop acoustic link, or has a deterministic path to an underwater node that can reach the surface node via one-hop acoustic link. For instance, in fig.2, Node 1 can reach a surface node via one-hop acoustic link, it is also the determistic relay of node 5 .
Keep in mind that our principle is to minimize the signaling overhead in acoustic subnetwork, in other words, to allocate the signaling traffic to the radio subnetwork as far as possible. To improve the efficiency of networking, it is necessary to apply a processing center that can control all the radio devices. In the next section we shall discuss some enabling technologies based on the new framework.

\section{ENABLING TECHNOLOGIES}

In the proposed radio-acoustic integrated network, the radio subnetwork has much higher capacity and lower transmission delay. From the viewpoint of the radio subnetwork, the acoustic subnetwork operates with extraordinary low speed. Hence the radio subnetwork has plenty of resources (bandwidth, time, power supply) to process the requests from the acoustic subnetwork. From the viewpoint of the acoustic subnetwork, it always get instantaneous response from the radio subnetwork. Hence, we argue that signaling overhead should be allocated to the radio subnetwork as far as possible. In the following we focus on the acoustic subnetwork and present the physicallayer, MAC-layer and network-layer techniques that can help moving the signaling overhead to the radio subnetwork.

\section{A. Multi-node Coordination}

The Coordination Multi-Point (CoMP) [9] technology is originally proposed to improve the system capacity of terrestrial wireless systems. In CoMP, it is assumed that the basestations (or access-points) are connected with high-speed cable or fibre and therefore they can instantaneously share the information. A group of spatially adjacent basestations then cooperate to form a virtual multiple antenna system, and they can cooperate in sending a signal to a group of terminals, or receiving a signal from it. In the proposed radioacoustic integrated network, the radio links have much higher transmission rate and hence can be considered as backbone link. Due to the broadcasting nature of acoustic signals, it is easy to cooperate several adjacent surface nodes and form virtual acoustic transferring array. As illustrated in fig.3, there are several feasible technologies that can be applied to improve the system capacity.

Coordinate uplink transmission. Fig.3(a) shows the coordination of uplink transmission, i.e., acoustic transmission from underwater to ocean surface. It is assumed that the uplink signals can naturally be overheard by receivers other than the intended receiver. Thus, if the receivers exchange their received signals and simultaneously perform the demodulating 


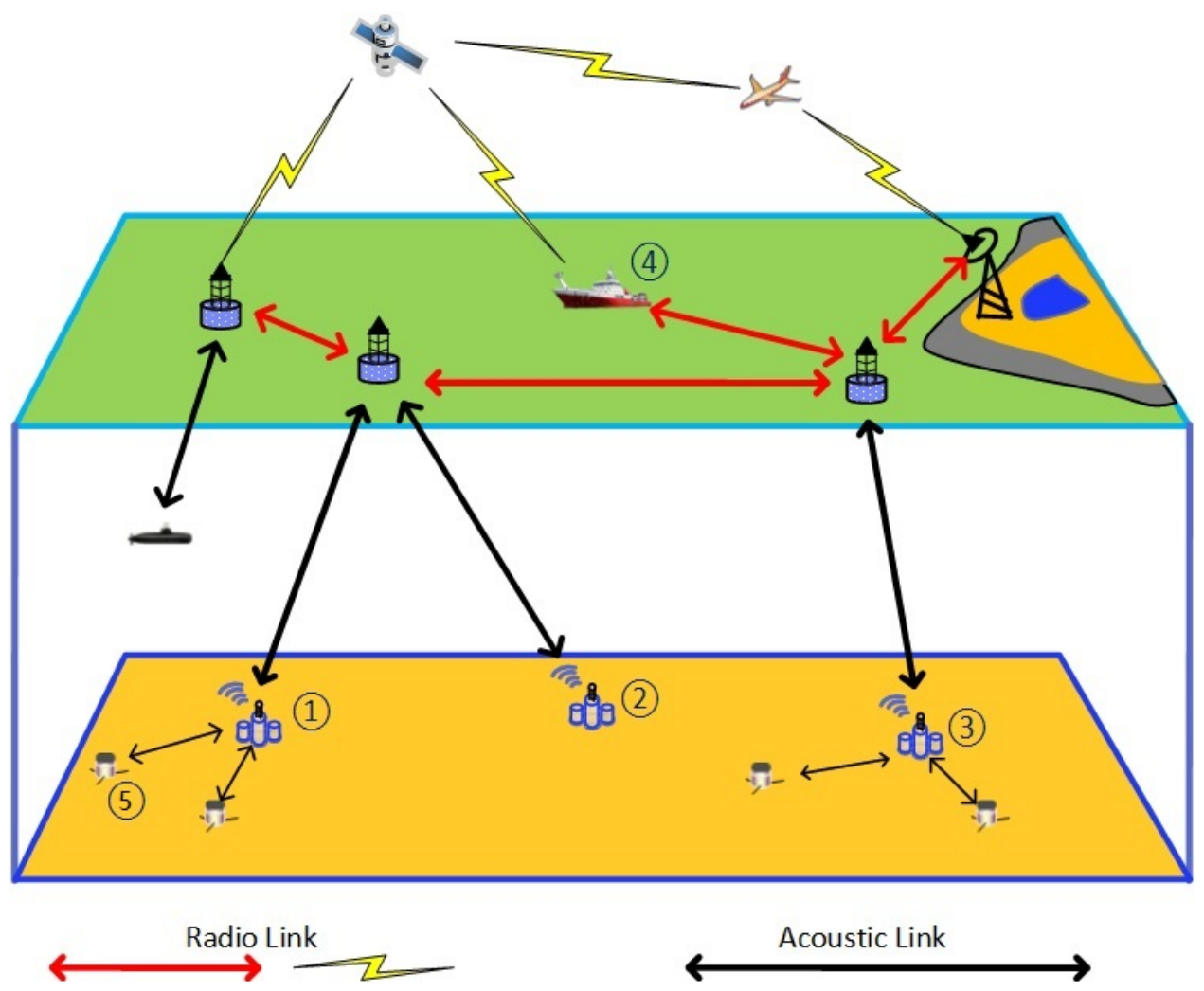

Fig. 2: Framework of radio-acoustic integrated networks.

algorithm, then the group of surface nodes can form a virtual receiver array and obtain the multiple receiver diversity.

Coordinate downlink transmission. There are two kinds of mechanisms to perform the downlink coordination(see fig.3(b)). When the group of surface nodes serve only one underwater node. The group of transmitters can simultaneously process the transmitted signal with certain space time coding techniques. When the group of surface nodes serve multiple underwater nodes, more complex techniques such as interference alignment and cancellation can be applied [10] [11]. This technique assumes accurate and detailed channel information of all downlinks are available to the intended receivers.

Coordinate point selection. In this technique the group of surface nodes are equipped with directed acoustic transducer, and hence they can serve different underwater nodes, as long as the transmission beams do not mutually interfere (shown in fig.3(c)).

Note that we can consider the surface gateway as the basestation and the underwater nodes as the user equipments. The above mentioned techniques then are similar to those which have been well investigated in terrestrial mobile systems [9], [12]. However, due to the specific features of acoustic channel, there are some limiting factors. First, the acoustic channels have extremely narrow bandwidth and large latency. Transmission techniques rely on the feedback of channel state, such as adaptive precoding/beamforming, may not be feasible. Second, due to the slow propagation speed of acoustic signals, the surface gateway may receive partially overlapped, or even non-overlapped data frames from geographically separated underwater nodes. In this case uplink coordinate reception is not applicable. New schemes should be applied to align the received data frame.

\section{B. Time alignted Scheduling}

The above Multi-node Coordination scheme allow concurrent transmission of the source nodes. Hence carrier sensing and handshaking are not necessary, which can save the time of waiting. Simple time-slotted architecture is applied to facilitate the Multi-node Coordination scheme. In radio subnetwork, due to the high propagation speed of radio signals, the time differences of multiple incoming signals at the receiver are usually within several symbol durations. Note that Multinode Coordination scheme does not require symbol-level synchronization [10]. Generally for radio network, concurrent transmission results in concurrent reception.

However, this is not the case in acoustic subnetwork. Due to the slow propagation speed of acoustic signals, timealigned transmission may not lead to time-aligned reception. As illustrated in fig.4(a), although the two sources send out the data packet simultaneously, at the receiver these two data packets partial overlap and introduce inter-slot interference. To tackle this problem, we assume that the transmission delays are known to the source nodes. The source nodes can schedule its transmission time such that the reception times approximately align at the receiver. We note that accurate alignment is not required because Multi-node Coordination scheme does not require symbol level synchronization. Moreover, we suggest a large slot duration (larger than the packer duration), such that inter-slot interference can be avoided, as illustrated in fig.4(b). 


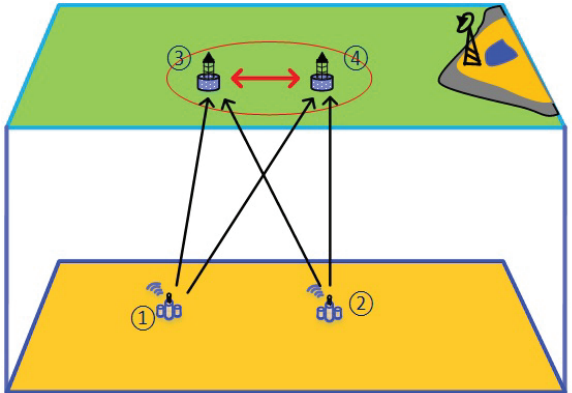

(a)

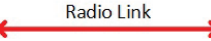

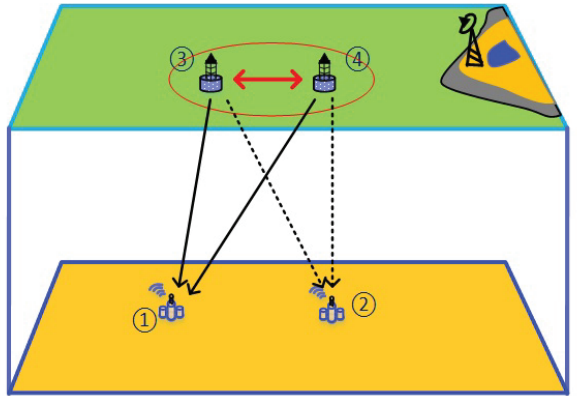

(b)

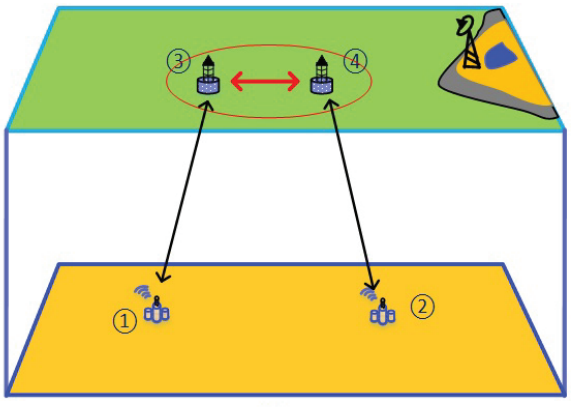

(c)

Fig. 3: Coordination of multiple surface nodes. (a) coordinative reception, $(b)$ coordinative transmission, $(c)$ coordinative access point selection.

In radio communication systems, the timing mismatch due to asynchronous transmission has been well investigated (e.g. see [13] and the references therein). It has been shown that when there is no inter-frame interference, the timing offsets due to asynchronous transmission can be considered as unknown coefficients to the signal model. Their impact on system spectral efficiency has been evaluated in theory and simulation [14]. It was shown in [14] that when the timing offsets can be properly estimated, the system spectral efficiency approaches the ideal performance without any timing offsets. Since acoustic communication systems have similar signal model with radio communication systems, we can extend existing research to acoustic communication system. Meanwhile we also need to cooperate with the slot scheduling scheme at the transmitters [15] to guarantee that there is not inter-frame interference.

\section{Coordinate Opportunistic Routing}

Traditional routing relay on network topology information. Such information usually is obtained by periodical signaling exchanges between neighbor nodes. Opportunistic Routing [16], however, takes advantage of the broadcast nature of the wireless medium. That is, instead of pre-selecting a specified relay node at each transmission, opportunistic routing broadcasts a data packet so that it is overheard by multiple neighbors which later form the candidate relays set. Then, the actual packet forwarder will be chosen from this set of candidates. Many factors make opportunistic routing the best routing alternative in mobile and dynamic wireless networks compared to the main traditional routing approaches.

Opportunistic routing has been applied in underwater acoustic networks. Since opportunistic routing protocols require many signaling exchanges among the sensor nodes in selecting the relay candidate set, they may lead to long delay in setting up routing due to the slow propagation speed of acoustic signals. In the proposed integrated network, we proposed a radio-acoustic coordinate scheme. As illustrated in fig.5, node 1 intends to transmit a data frame to node 2 . It directly sends the data frame to the surface nodes. Based on the multi-node coordination scheme, naturally there is a group of surface nodes that receive the data frame. This group of nodes then forward the data frame according to the opportunistic routing schemes, until the data frame reaches the group of nodes that directly reach the underwater destination node.

In the proposed scheme, routing is not required in underwater acoustic subnetwork. The signaling overhead is well allocated to the radio subnetwork.

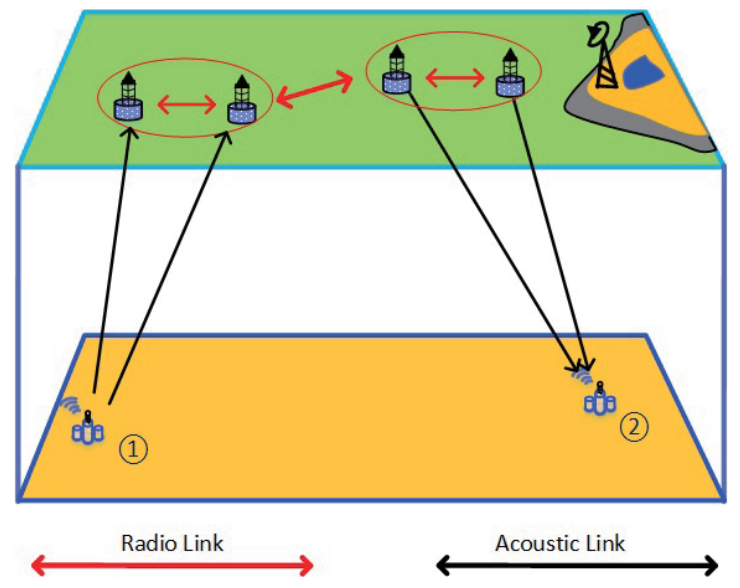

Fig. 5: Surface opportunistic routing assisted data transmission.

\section{Dynamic Topology Control}

Mobile nodes have been widely applied in terrestrial radio networks and underwater acoustic networks, as data sinks or relays to maintain the network connectivity. In [17] unmanned aerial vehicles (UAVs) were applied as data acquisition platforms to collect data of multiple water surface nodes. In [18], autonomous underwater vehicles were applied as data relays. Similarly, we propose to use unmanned surface vessel as mobile nodes to cover the connection hope in the network, and mentain the network connectivity, as illustrated in fig.6.

\section{DISCUSSION AND CONCLUSION}

In this paper, a novel radio-acoustic integrated network model is proposed to extend the internet coverage under ocean surface. Based on the fact that radio links are far more efficient than acoustic links, the key principle of radio-acoustic integrated network is that the throughput overhead of signaling 


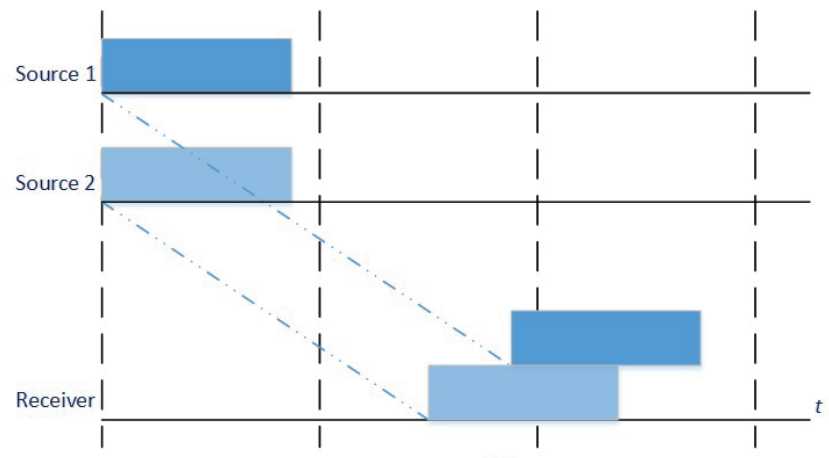

(a)

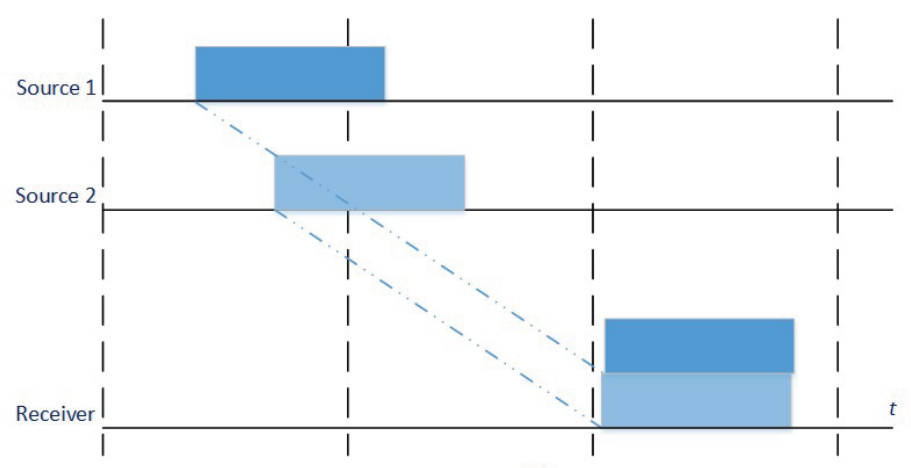

(b)

Fig. 4: Slot scheduling mechanism, (a) time-aligned transmission, (b) time-aligned reception.

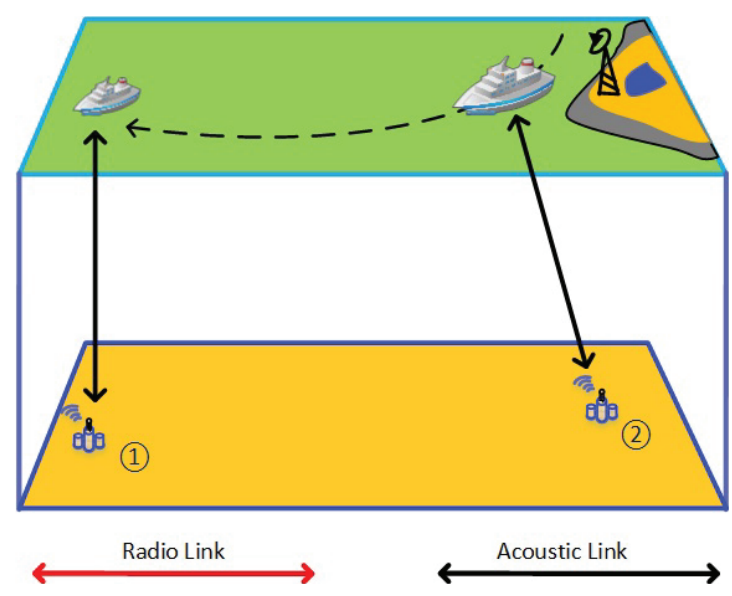

Fig. 6: Automatic surface vessel as relay to assist underwater data transmission

exchange should be allocated to the radio subnetwork as far as possible. Several technologies that can help moving the signal overhead to radio subnetwork are presented. Other important research topics for future work may include

Time synchronization. Time synchronization is crucial for time-slotted network. The surface nodes can implement time synchronization with the global positioning system(GPS). Since underwater nodes cannot receive the GPS signal, it is important to synchronize the underwater nodes with the aid of surface nodes.

Localization. In multi-node coordination, the group of surface nodes need to known the locations of underwater nodes, so as to determine their serving surface nodes.

Delay and channel estimation. To perform time alignment at the receiver, it is necessary to known the channel propagation delay. Meanwhile, channel information is important in performing interference alignment precoding.

To facilitate the deployment of radio-acoustic integrated network, these research topics require further investigation in the future work.

\section{ACKNOWLEDGEMENT}

This work was supported in part by the National Key Research and Development Program of China under grant
2020YFB1807700, in part by the National Natural Science Foundation of China under Grants U1701265, and in part by Key Program of Marine Economy Development, Department of Natural Resources of Guangdong Province under Grant YZRZH[2020]009.

\section{REFERENCES}

[1] H. Yao, L. Wang, X. Wang, Z. Lu, and Y. Liu, "The space-terrestrial integrated network: An overview," IEEE Communications Magazine, vol. 56, no. 9, pp. 178-185, 2018.

[2] L. Yin, C. Jiang, C. Jiang, and Y. Qian, "Collaborative spectrum managements and sharing in coordinated space, terrestrial and ocean networks," IEEE Network, vol. 34, no. 1, pp. 182-187, 2020.

[3] S. Jiang, "Networking in oceans: A survey," ACM Computing Surveys, vol. 54, no. 1, pp. 1-33, 2021.

[4] W. Chien, C. Lai, M. S. Hossain, and G. Muhammad, "Heterogeneous space and terrestrial integrated networks for iot: Architecture and challenges," IEEE Network, vol. 33, no. 1, pp. 15-21, 2019.

[5] S. Basagni, M. Conti, S. Giordano, and I. Stojmenovic, Advances in Underwater Acoustic Networking. John Wiley \& Sons, Inc., 2013.

[6] Q. Guan, W. Chen, H. Yu, and F. Ji, "Acoustic-radio cooperative marine information network (in chinese)," Telecommunication Science, vol. 34, no. 6, pp. 21-28, 2018.

[7] M. Stojanovic and J. Preisig, "Underwater acoustic communication channels: Propagation models and statistical characterization," IEEE Communications Magazine, vol. 47, no. 1, pp. 84-89, 2009.

[8] X. Zhong, F. Ji, F. Chen, Q. Guan, and H. Yu, "A new acoustic channel interference model for 3-d underwater acoustic sensor networks and throughput analysis," IEEE Internet of Things Journal, vol. 7, no. 10, pp. 9930-9942, 2020.

[9] J. Lee, Y. Kim, H. Lee, B. L. Ng, D. Mazzarese, J. Liu, W. Xiao, and Y. Zhou, "Coordinated multipoint transmission and reception in lteadvanced systems," IEEE Communications Magazine, vol. 50, no. 11, pp. 44-50, 2012.

[10] G. Shyamnath, P. S. David, and K. Dina, "Interference alignment and cancellation," ACM SIGCOMM Computer Communication Review, 2009.

[11] F. Liu, S. Jiang, S. Jiang, and C. Li, "Dof achieving propagation delay aligned structure for $\mathrm{k} \times 2 \mathrm{x}$ channels," IEEE Communications Letters, vol. 21, no. 4, pp. 897-900, 2017.

[12] D. N. Nguyen and M. Krunz, "Cooperative mimo in wireless networks: recent developments and challenges," Network IEEE, vol. 27, no. 4, pp. 48-54, 2013.

[13] X. Zou, B. He, and H. Jafarkhani, "An analysis of two-user uplink asynchronous non-orthogonal multiple access systems," IEEE Transactions on Wireless Communications, vol. 18, no. 2, pp. 1404-1418, 2019.

[14] F. Yang, X. Zhang, P. Cai, and X. Luo, "Massive mimo performance with timing frequency errors," in 2017 IEEE Global Conference on Signal and Information Processing (GlobalSIP), 2017, pp. 269-273.

[15] M. Liu, X. Zhuo, Y. Wei, Y. Wu, and F. Qu, "Packet-level slot scheduling mac protocol in underwater acoustic sensor networks," IEEE Internet of Things Journal, pp. 1-1, 2021. 
[16] N. Chakchouk, "A survey on opportunistic routing in wireless communication networks," IEEE Communications Surveys Tutorials, vol. 17, no. 4, pp. 2214-2241, 2015.

[17] Q. Wang, H. Dai, Q. Wang, M. K. Shukla, W. Zhang, and C. G. Soares, "On connectivity of uav-assisted data acquisition for underwater internet of things," IEEE Internet of Things Journal, vol. 7, no. 6, pp. 5371-5385, 2020.

[18] X. Zhuo, M. Liu, Y. Wei, G. Yu, F. Qu, and R. Sun, "Auv-aided energyefficient data collection in underwater acoustic sensor networks," IEEE Internet of Things Journal, vol. 7, no. 10, pp. 10010-10 022, 2020. 